\title{
miR-375 inhibits the invasion and metastasis of colorectal cancer via targeting SP1 and regulating EMT-associated genes
}

\author{
FENGYUN CUI $^{1,2}$, SHUYANG WANG ${ }^{1}$, IWENG LAO ${ }^{1}$, CHUNXIAN ZHOU $^{1}$, HUI KONG $^{1}$, NAYIMA BAYAXI ${ }^{1}$, \\ JIALI LI ${ }^{1}$, QI CHEN ${ }^{1}$, TENGFANG ZHU ${ }^{1}$ and HONGGUANG ZHU ${ }^{1,3,4}$ \\ ${ }^{1}$ Department of Pathology, School of Basic Medical Sciences, Fudan University, Shanghai 200032; \\ ${ }^{2}$ Division of Surgical Pathology, Shandong Shengli Hospital, Shandong University, Shanghai 250021; \\ ${ }^{3}$ Division of Surgical Pathology, Huashan Hospital, Fudan University, Shanghai 200040; \\ ${ }^{4}$ Institutes of Biomedical Sciences, Fudan University, Shanghai 200032, P.R. China
}

Received January 11, 2016; Accepted March 4, 2016

DOI: $10.3892 /$ or.2016.4834

\begin{abstract}
Accumulating evidence has shown that aberrantly expressed microRNAs (miRNAs) are associated with tumor development and progression. Our previous study found that microRNA-375 (miR-375) was downregulated in colorectal cancer (CRC), but little is known concerning the role of miR-375 and the related mechanism in CRC development. The proliferation, invasion and migration effects were investigated by Cell Counting Kit-8 (CCK-8), colony formation and Transwell assays with or without Matrigel. In addition, candidate target genes were screened and validated by luciferase reporter and western blot assays. In addition, western blot analysis was performed to explore the molecular mechanisms associated with epithelial-mesenchymal transition (EMT). It was found that miR-375 inhibited proliferation, invasion and migration in DLD1 and HCT8 cells. In addition, miR-375 negatively regulated $\mathrm{Sp} 1$ transcription factor (SP1) protein by directly binding to the 3'-untranslated region (3'-UTR). Furthermore, it was found that miR-375 regulated matrix metalloproteinase 2 (MMP2) and EMT-associated genes, E-cadherin, vimentin, snail, $\mathrm{N}$-cadherin and $\beta$-catenin. In conclusion, miR-375 inhibited the proliferation, invasion and migration by directly targeting SP1 and regulating MMP2 and EMT-associated genes.
\end{abstract}

\section{Introduction}

Colorectal cancer (CRC) is the third most common malignant cancer diagnosed worldwide (1), and ranks as the second leading cause of cancer-related deaths in developed countries (2). Approximately $90 \%$ of CRC-related mortalities are

Correspondence to: Dr Hongguang Zhu, Department of Pathology, School of Basic Medical Sciences, Fudan University, 138 Yi Xue Yuan Road, Shanghai 200032, P.R. China

E-mail: hongguang_701@163.com

Key words: CRC, miR-375, metastasis, SP1, EMT a result of metastases (3). Metastasis is a complex, multi-step process whereby tumor cells first invade the surrounding tissues and intravasate into the vasculature, then translocate through the systemic circulation, and thus extravasate into the parenchyma of distant tissues, e.g. liver and lungs, eventually, establishing micrometastases and forming macroscopic secondary tumors (4).

Although much progress has been made in the identification and characterization of the genetic and epigenetic changes involved in CRC metastasis $(5,6)$, the underlying mechanisms remain largely unclear. The search for predictive markers for CRC metastasis remains a priority, as it is the major cause of the high mortality rate. Recently, microRNAs (miRNAs) have become a hot spot due to their significant role in the regulation of gene expression. miRNAs are 22 -nucleotide conserved endogenous non-coding single-stranded RNA molecules (7), which bind to the 3'-untranslated region (3'-UTR) of target mRNAs and regulate the stability and translation of mRNAs, resulting in either inhibition of translation or degradation of target mRNAs $(8,9)$. They are involved in a wide spectrum of biological processes, such as proliferation, metabolism, cellular differentiation and apoptosis $(10,11)$. To date, a number of miRNAs have been found to be associated with cancer development and progression, including CRC. Among them, miR-375 is proven to be involved in early invasive CRC through downregulation of its expression (12-14); however, the functional role of miR-375 in CRC needs further investigation (15). In the present study, we attempted to reveal the mechanisms of miR-375 underlying the biological behavior of CRC. We investigated the biological functions of proliferation and invasion/migration in CRC cells and identified the target gene in order to explore the possible molecular mechanism involving $\mathrm{CRC}$ development, in hope of identifying a new predictor for prognosis or new target for diagnosis and therapy.

\section{Materials and methods}

Cell lines and transfection. DLD1 and HCT8 cells stored in our laboratory were maintained in RPMI-1640 medium, supplemented with $10 \%$ fetal bovine serum (FBS) (both from Gibco, Grand Island, NY, USA) at $37^{\circ} \mathrm{C}$ under $5 \%$ 
$\mathrm{CO}_{2}$. We had entrusted Beijing Microread Genetics Co., Ltd., Beijing, China for the identification of the 2 cell lines. The authentication reports (data not shown) showed no cross-contamination of other human cell lines, and 100\% matched cell lines are found in ATCC banks with the DLD1 and HCT8 cell lines. Precursor miRNA (pre-miR-375) and precursor negative control (pre-neg) (Applied Biosystems, Foster City, CA, USA) were transfected into the two cell lines using Lipofectamine 2000 (Invitrogen, Carlsbad, CA, USA) according to the manufacturer's instructions. Additionally, following this method, the interfering RNA of sp1 messenger RNA (siSP1) and the negative control (si-neg) were transfected into the HCT8 cells.

RNA extraction and quantitative RT-PCR ( $q R T-P C R)$. Total RNA of the cells was extracted using mirVana isolation kit according to the manufacturer's instructions (Applied Biosystems). For validation of miRNA and target gene mRNA expression, quantitative reverse transcriptase-polymerase chain reaction (qRT-PCR) using TaqMan miRNA assays and quantitative RT-PCR using SYBR-Green PCR Master Mix kit (Applied Biosystems) were performed according to the manufacturer's instructions. The expression level of U47 small nuclear RNA was used for miRNA, and GAPDH was used for mRNA as the endogenous controls. All assays were carried out in triplicate.

Cell colony formation assay. For the colony formation assay, $1 \times 10^{3}$ cells were plated in 6 -well plates for each well in medium with $20 \%$ FBS for $72 \mathrm{~h}$, and then fixed and stained with crystal violet. Then, the colonies were photographed and counted under a microscope. All experiments were performed in 3 replicates and were repeated 3 times, independently.

Cell Counting Kit-8 (CCK-8) assay. DLD1 $\left(2 \times 10^{3}\right)$ and HCT8 cells $\left(3 \times 10^{3}\right)$ were suspended in RPMI-1640 medium $(100 \mu \mathrm{l})$ containing 10\% FBS and cultured into 96-well plates overnight, and then transfected with pre-miR-375 and pre-neg. Cell proliferation was determined using CCK- 8 assay at $0,1,2$ and 3 days after transfection, respectively, and the absorbance of the samples was measured with a spectrophotometer reader at $450 \mathrm{~nm}$.

Cell migration and invasion assays. DLD1 $\left(2 \times 10^{5}\right)$ and HCT8 cells $\left(2 \times 10^{5}\right)$ were cultured into 6 -well plates, at $24 \mathrm{~h}$ after transfection of pre-miR-375 and pre-neg, as previously described. Migration and invasion assays were performed both using 24-well Transwell migration chambers with 8- $\mu 1$ pore size (Corning Costar, Inc., Corning, NY, USA). DLD1 (1x10 $)$ and HCT8 cells $\left(5 \times 10^{4}\right)$ suspended in $100 \mu \mathrm{l}$ corresponding culture medium without FBS were loaded into the top chamber, and the bottom chamber contained $600 \mu$ l medium with $20 \%$ FBS. For the migration assay, the cells were allowed to migrate for $12 \mathrm{~h}$. In addition, for the invasion assay, Transwell wells were pre-coated with Matrigel, and the cells were allowed to invade for $48 \mathrm{~h}$. When both assays were stopped, the cells that migrated or invaded into the bottom chamber were fixed with methanol for $5 \mathrm{~min}$, and then stained with crystal violet $(0.05 \%)$ for $4 \mathrm{~min}$. The cells that migrated or invaded were photographed and counted under a microscope. In addition, the migration and invasion rates were assessed by the formula: (motile cells transfected with pre-miR-375/motile cells transfected with pre-neg). All experiments were independently repeated in triplicate.

Luciferase reporter assay. To elucidate the molecular mechanisms involved in the effects of miR-375 on CRC cells, putative miR-375 target genes were predicted through the gateway miRecords (http://mirecords.biolead.org/). To improve the accuracy of the prediction, the genes that were predicted by at least 4 of 11 databases (DIANA-microT, MicroInspector, miRanda, MirTarget2, miTarget, NBmiRTar, PicTar, PITA, RNA22, RNAhybrid and TargetScan) were selected as candidate targets. Wild and mutant putative targets of the Sp1 transcription factor (SP1) 3'-UTR (Fig. 3A) were cloned into pmiReport vector (Ambion, Carlsbad, CA, USA). 293T cells $\left(2 \times 10^{4}\right)$ were co-transfected with $500 \mathrm{ng}$ of wild or mutant constructs of the SP1 3'-UTR with pre-miR-375 or pre-neg. Each sample was cotransfected with $50 \mathrm{ng}$ of pRL-TK plasmid expressing Renilla luciferase to monitor the transfection efficiency. A luciferase activity assay was performed $48 \mathrm{~h}$ after transfection with the dual-luciferase reporter assay system. The relative luciferase activity was normalized to Renilla luciferase activity.

Western blot analysis. DLD1 and/or HCT8 cells $\left(1 \times 10^{5}\right)$ were incubated in 6-well plates for $72 \mathrm{~h}$. Then, cell proteins were harvested and homogenized with lysis buffer (Tiangen, Shanghai, China). Proteins from the cells were resolved by $10 \%$ SDS-PAGE gel and transferred to a nitrocellulose membrane (Millipore, Billerica, MA, USA). The membrane was incubated with primary antibody at $4^{\circ} \mathrm{C}$ overnight, then the secondary antibody for $1 \mathrm{~h}$ at $37^{\circ} \mathrm{C}$ and finally visualized by enhanced chemiluminescence (ECL; Thermo Fisher Scientific, Inc., Waltham, MA, USA). The antibodies used were: SP1 (1:2,000; Abcam); matrix metalloproteinase 2 (MMP2) $(1: 1,000)$, E-cadherin $(1: 2,000)$, vimentin $(1: 500)$, snail $(1: 1,000), \beta$-catenin $(1: 1,000)$ [all from Cell Signaling Technology (CST), Beverly, MA, USA]; $\beta$-actin $(1: 1,000)$ and GAPDH $(1: 2,000)$ (Santa Cruz Biotechnology, Inc., Santa Cruz, CA, USA).

Immunofluorescence analysis. $\mathrm{HCT} 8$ cells $\left(5 \times 10^{4}\right)$ were seeded into 6-well plates containing 13-mm collagen (Sigma, St. Louis, MO, USA)-coated coverslips. After $12 \mathrm{~h}$, the cells were transfected with pre-miR-375 and pre-neg. Then, for another $48 \mathrm{~h}$, the cells on the coverslips were washed using cooled PBS on ice twice with PBS for 5 min. Primary antibodies were diluted in $1 \%(\mathrm{w} / \mathrm{v}) \mathrm{BSA} / \mathrm{PBS}$ and applied to the coverslips and incubated on ice for $1 \mathrm{~h}$. After 3 washes with PBS, coverslips were incubated with cy3-conjugated secondary antibody diluted in $1 \%(\mathrm{w} / \mathrm{v}) \mathrm{BSA} / \mathrm{PBS}$ on ice for $30 \mathrm{~min}$. The cells were washed twice with PBS before coverslips were mounted onto glass slides using Gelvatol mounting medium. Slides were viewed using an Olympus IX71 microscope fitted with a U-RFL-T fluorescent lamp, and images were captured and analyzed using DP Controller software (Olympus Corporation, Tokyo, Japan).

Expression of miR-375 in metastatic CRC tissues. Fifteen snap-frozen tumor tissues from 5 patients who were surgi- 

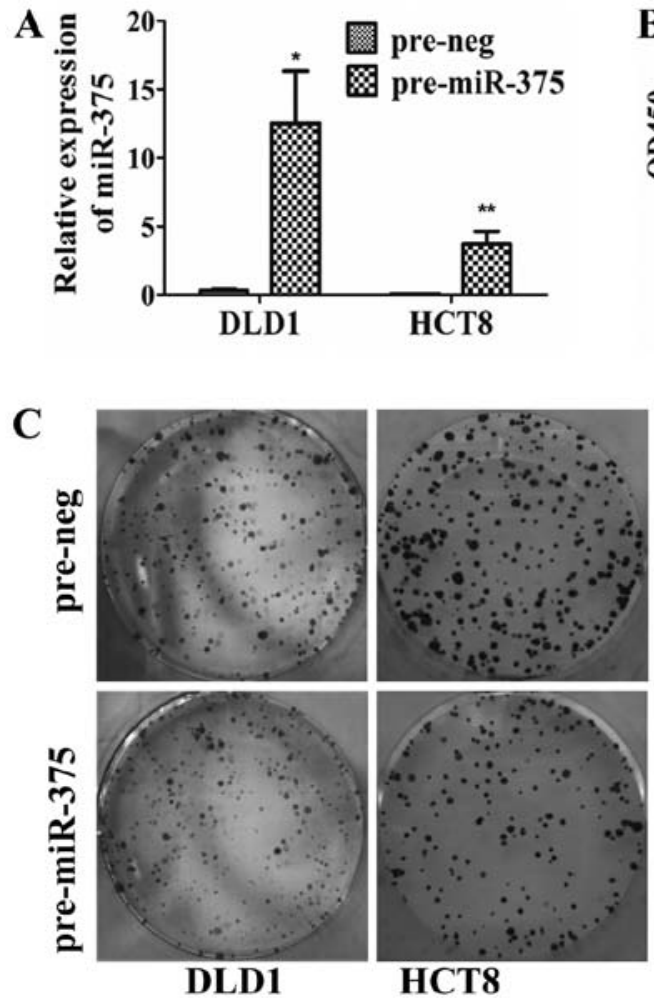
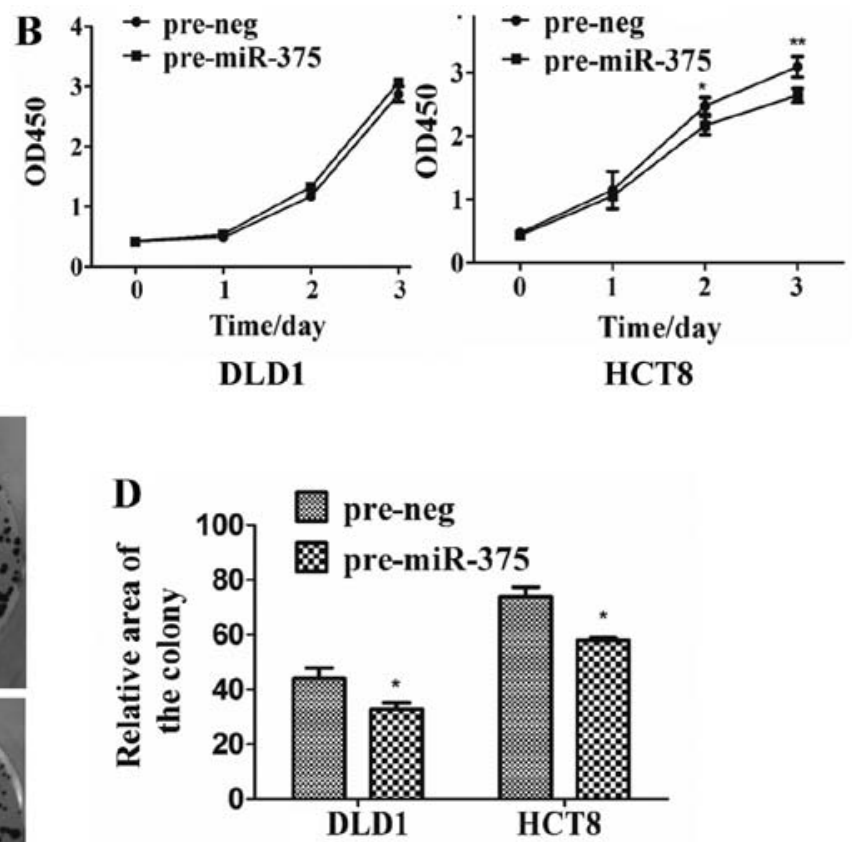

Figure 1. miR-375 inhibits CRC cell proliferation. (A) miR-375 was overexpressed in DLD1 and HCT8 cells transfected with pre-miR-375 compared with its expression in cells transferred with pre-neg. (B) By CCK-8 analysis, overexpression of miR-375 reduced the growth of HCT8 cells, but not obviously in the DLD1 cells. (C and D) Colony formation assay showed that overexpression of miR-375 decreased the number and mean area of the colonies in the DLD1 and HCT8 cells.

cally resected at Shanghai Huashan Hospital in 2008 were obtained. Each group included primary cancer (T), lymphatic metastasis $(\mathrm{N})$ and liver metastasis tissues $(\mathrm{M})$ from the same patient. The present study was approved by the Institutional Review Board of Shanghai Medical College, Fudan University and informed consent was obtained from all patients. The RNA extraction and qRT-PCR protocols are referred to as above.

Statistical analysis. The data are expressed as mean \pm SD. Student's t-test was used to compare test samples with the controls. Two-way analysis of variance was used to compare differences among three or more experimental groups. Statistical analyses were performed using SPSS 11.0 software. $\mathrm{P}<0.05$ was considered to indicate a statistically significant result.

\section{Results}

miR-375 inhibits the proliferation of CRC cells. To further determine the role of miR-375 in CRC cells, expression of miR-375 was upregulated in DLD1 cells by 40.9 -fold $(\mathrm{P}=0.0040)$ and in HCT8 cells by 89.2 -fold $(\mathrm{P}=0.0052$ ) (Fig. $1 \mathrm{~A})$ compared with the negative control. Overexpression of miR-375 repressed the proliferation of CRC cells as detected by CCK-8 and colony formation assays. CRC HCT8 cells transfected with pre-miR375 were observed to grow more slowly by $18.8 \%(\mathrm{P}=0.0096)$ on day 3 (Fig. 1B). The cell colony average area in the DLD1 cells transfected with pre-miR-375 was reduced by $25 \%(\mathrm{P}=0.0201)$, and by $21.5 \%$ in the HCT8 cells ( $\mathrm{P}=0.0136)$ (Fig. $1 \mathrm{C}$ and $\mathrm{D})$.
miR-375 inhibits the migration and invasion of CRC cells. To validate the involvement of miR-375 in metastasis, migration and invasion assays were performed in the DLD1 and HCT8 cells. In the migration assay, the numbers of migrating cells transfected with pre-miR-375 were significantly reduced, and the migration rate decreased by $33 \%$ in the DLD1 cells ( $\mathrm{P}=0.0138)$, and $36 \%$ in the HCT8 cells $(\mathrm{P}=0.0140)$ compared with the pre-neg groups (Fig. 2A). In the invasion assay, the numbers of invading cells transfected with pre-miR-375 were significantly reduced, and the invasion rate decreased by $46 \%$ in the DLD1 cells $(\mathrm{P}=0.0479)$, and $52.3 \%$ in the HCT8 cells $(\mathrm{P}=0.0422)$ compared with the pre-neg groups (Fig. 2B).

SPI is identified as a target of $m i R-375$. SP1 was found to be one of the candidate target genes, which bears a miR-375 binding sites in its 3'-UTR. To determine whether miR-375 can regulate the expression of SP1, luciferase reporter assay was performed with a vector containing the wild-type (WT) or mutant (MUT) (Fig. 3A) putative SP1 3'-UTR target site downstream of the luciferase reporter pMIR-REPORT vector. When pre-miR-375 was co-transfected, the relative luciferase activity of the reporter containing WT-3'-UTR was significantly suppressed by $28.5 \%(\mathrm{P}=0.0203)$ compared with that of the reporter containing NC-3' UTR. In contrast, the luciferase activity of the reporter containing MUT-3'-UTR was unaffected by simultaneous transfection with NC-3'-UTR (Fig. 3B). Then, western blotting and qRT-PCR results showed that overexpression of miR-375 decreased the SP1 expression at the protein (Fig. 3D) but not the messenger RNA level (Fig. 3C). 

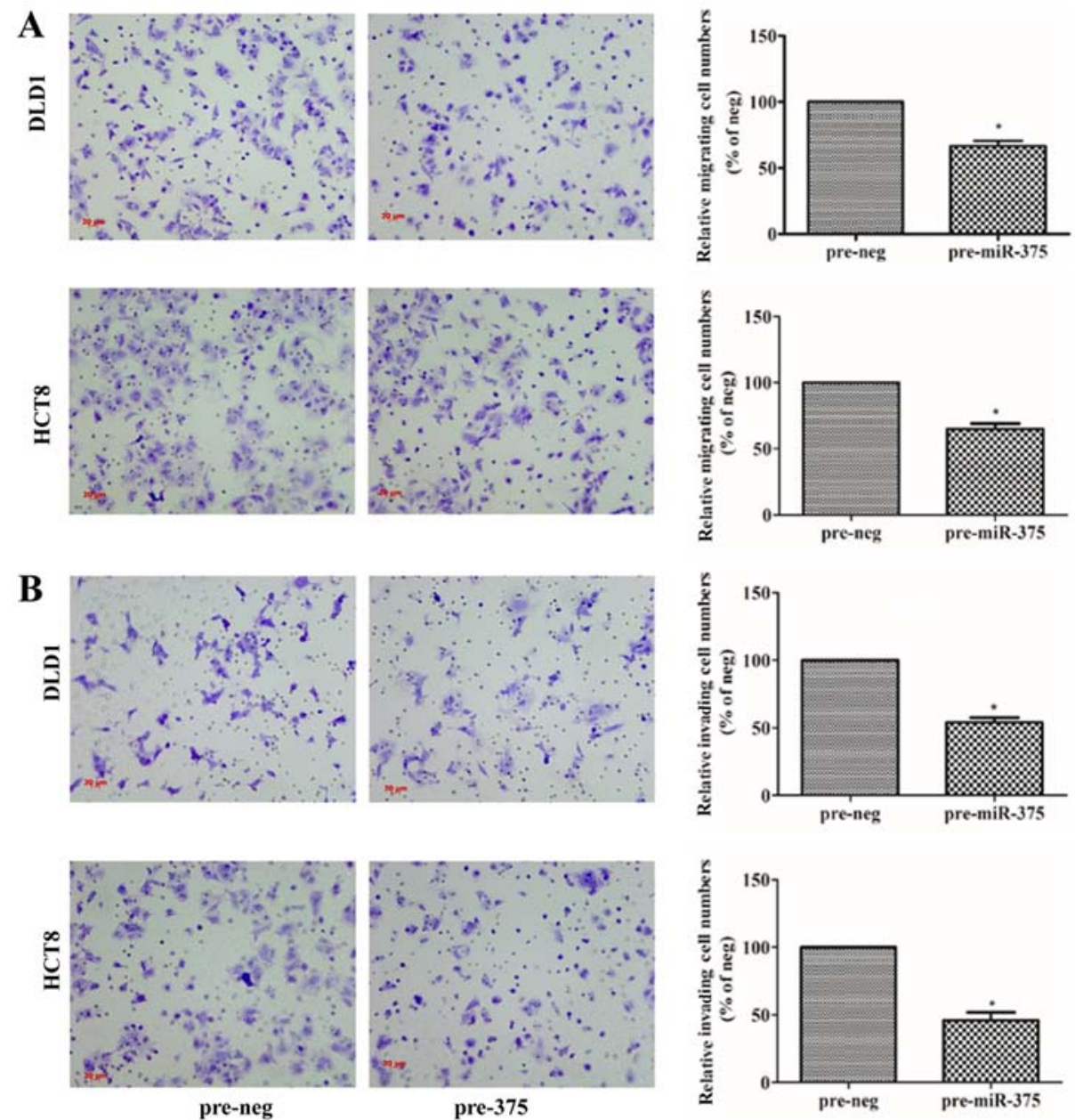

Figure 2. Overexpression of miR-375 inhibits CRC cell invasion and migration. (A) The migration of the DLD1 and HCT8 cells transfected with pre-miR-375 was reduced compared with that noted in the cells transfected with pre-neg. (B) Invasion of the DLD1 and HCT8 cells transfected with pre-miR-375 was reduced compared with that noted in the cells transfected with pre-neg. The motile cells were stained with Gentian violet, and photographed at magnification (x200). The migration or invasion rates were calculated as: Motile cells transfected with pre-miR-375/motile cells transfected with pre-neg.

A
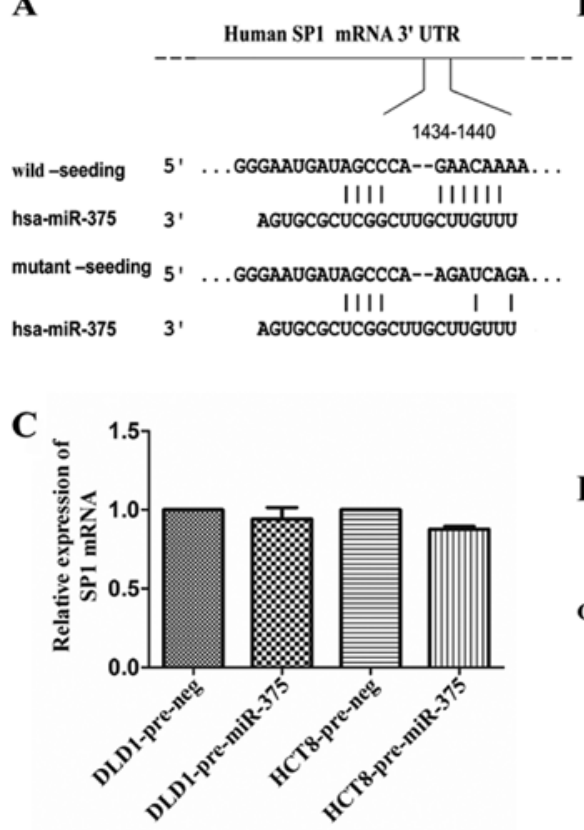

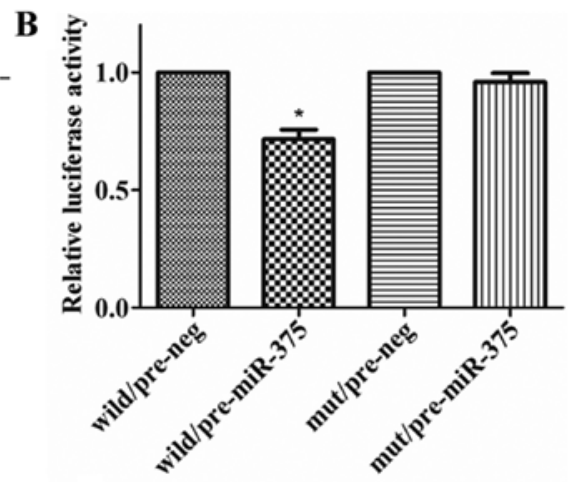

D

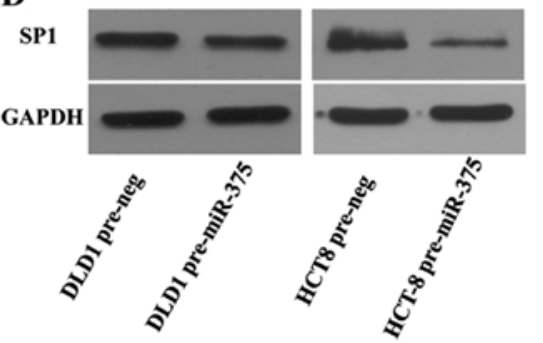

Figure 3. Prediction and verification of the target gene of miR-375. (A) The seeding sequence of the miR-375 target on the 3'-UTR of SP1. (B) Luciferase reporter assay analysis. Overexpression of miR-375 reduced the luciferase activity of wild-type 3'-UTR-SP1, but not the mutant 3'-UTR-SP1. (C and D) Upregulation of miR-375 decreased the SP1 protein level but not the SP1 mRNA in the CRC cells. 
A

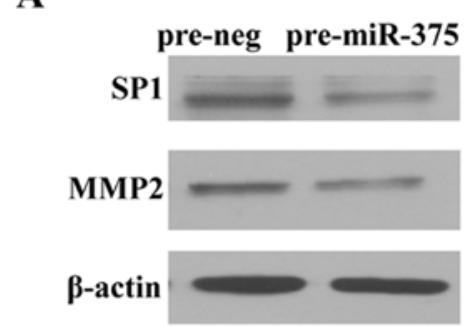

C

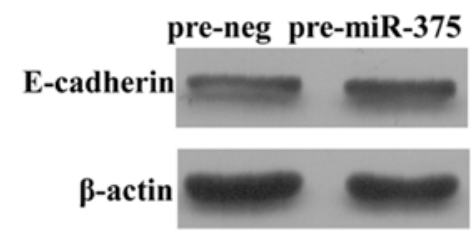

E

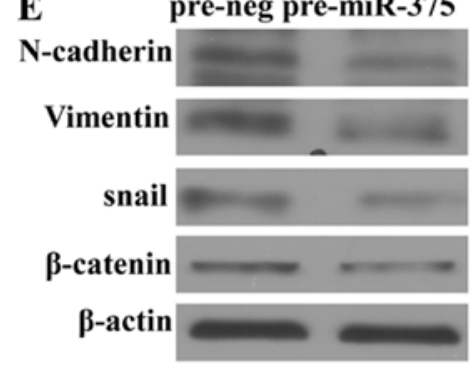

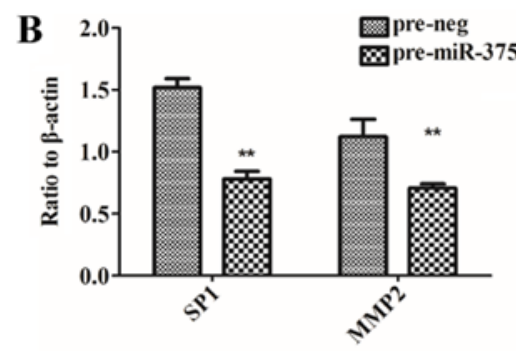

D

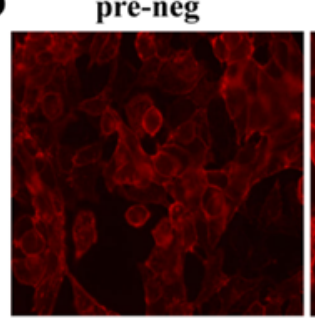

pre-miR-375

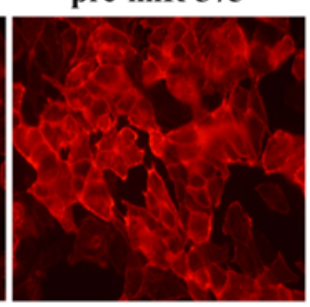

F

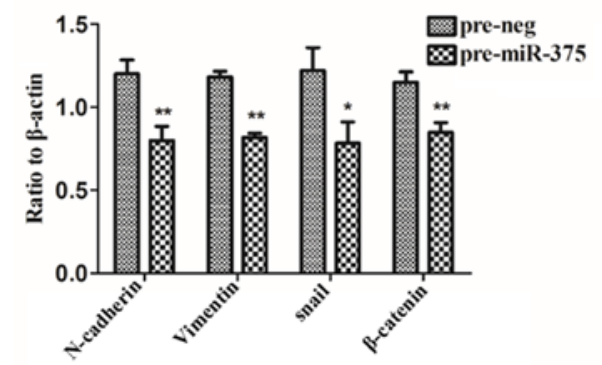

Figure 4. miR-375 regulates MMP2 and EMT-associated genes. (A and B) Western blot analysis showed that overexpression of miR-375 reduced SP1 and MMP2 protein but (C) did not increase the whole cell E-cadherin protein obviously. (D) Immunofluorescence analysis showed that upregulation of miR-375 increased E-cadherin on the HCT8 cell membrane, and (E and F) inhibited expression of EMT-associated proteins: N-cadherin, vimentin, snail and $\beta$-catenin. ${ }^{*} \mathrm{P}<0.05$, ${ }^{* * *} \mathrm{P}<0.01$.

A
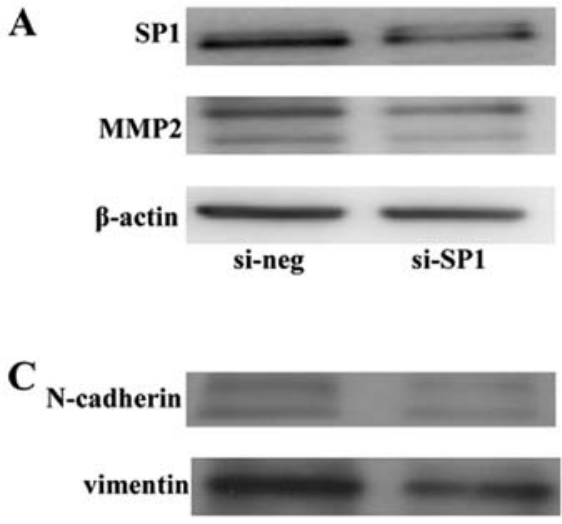

$$
\text { snail }
$$

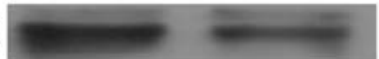

$\beta$-catenin

$\beta$-actin
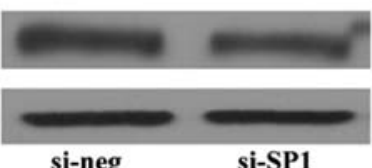

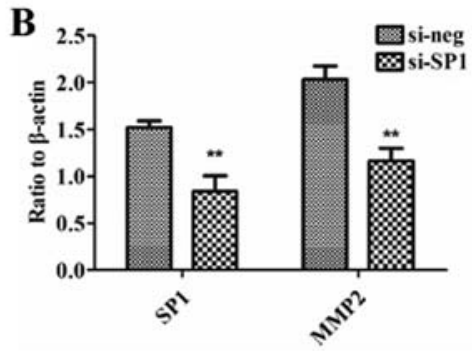

D

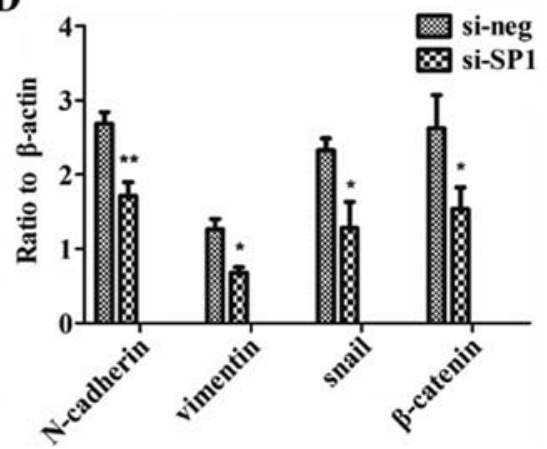

Figure 5. Downregulation of SP1 protein by siSP1 regulates MMP2 and EMT-associated genes. Transfection of siSP1 in HCT8 cells reduced SP1 protein successfully, and at the same time reduced MMP2 (A and B), and also decreased the expression of EMT-associated genes in the HCT8 cells $(\mathrm{C}$ and $\mathrm{D})$. ${ }^{*} \mathrm{P}<0.05,{ }^{* *} \mathrm{P}<0.01$.

miR-375 is involved in the epithelial-mesenchymal transition (EMT) of CRC cells via regulation of SP1. To further explore the molecular mechanisms of how miR-375 regulates migration and invasion development, we tested whether miR-375 regulates the expression of MMP-2 and EMT-related genes through SP1. The data showed that overexpression of miR-375 in the HCT8 
A

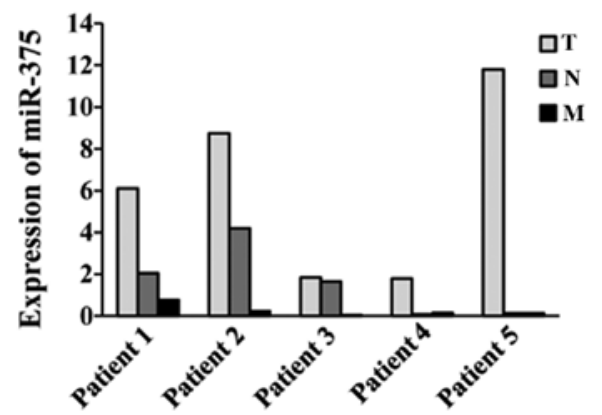

$\mathrm{B}$

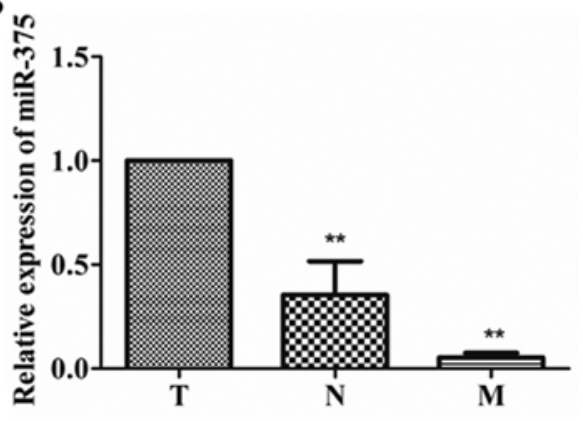

Figure 6. qRT-PCR analysis of miR-375 in metastastic CRC tissues. (A) Expression of miR-375 in 15 samples from 5 patients: T, primary CRC; N, lymphatic metastasis and M, liver metastasis samples. (B) miR-375 was significantly downregulated in $\mathrm{N}$ and $\mathrm{M}$ compared with $\mathrm{T}$.

cells reduced SP1 and MMP2 protein (Fig. 4A and B). In addition, immunofluorescence assay showed that upregulation of miR-375 predominantly increased E-cadherin protein on the HCT8 cell membrane (Fig. 4D), even though the whole protein of E-cadherin did not increase obviously (Fig. 4C). Upregulation of miR-375 reduced $\mathrm{N}$-cadherin, vimentin, snail and $\beta$-catenin proteins in the HCT8 cells $(\mathrm{P}<0.05$, $\mathrm{P}<0.01$ ) (Fig. 4E and F).

Downregulation of SP1 by siSP1 regulates $M M P 2$ and EMT-associated genes. To further explore whether SP1 regulates MMP2 and EMT-associated genes, SP1 was interfered by siSP1 in the HCT8 cells. In addition, the results showed that interference with siSP1 reduced SP1 protein, and at the same time reduced expression of MMP2 (Fig. 5A and B) and EMT-associated genes (Fig. 5C and D) $(\mathrm{P}<0.05, \mathrm{P}<0.01)$.

miR-375 is downregulated in metastatic CRC. Expression of miR-375 in primary cancer $(\mathrm{T})$, lymphatic metastasis $(\mathrm{N})$ and liver metastasis tissues (M) were examined. In addition, miR-375 was downregulated in all the 5 lymphatic metastasis and liver metastasis tissues compared with primary cancer tissues (Fig. 6A). In addition, miR-375 was significantly downregulated in lymphatic metastasis by $65 \%(\mathrm{P}=0.0163)$ and liver metastasis tissues by $94.7 \%(\mathrm{P}=0.0000)$ compared with primary cancer (Fig. 6B).

\section{Discussion}

MicroRNAs (miRNAs) are considered as potential specific biomarkers and play important roles in the diagnosis, progression and prognosis of many types of cancers (16-18). miR-375 was first identified as a pancreatic islet-specific miRNA regulating insulin secretion $(19,20)$. Current studies show that miR-375 is decreased in liver, lung and gastric cancer, and could participate in the regulation of cancer development as a tumor-suppressor gene by targeting YAP1 and JAK2 $(21,22)$, but its association with colorectal cancer (CRC) development was not carefully investigated. Our previous study showed that miR-375 was weakly expressed in CRC, and coupled with another two miRNAs could distinguish early invasive CRC from high-grade intraepithelial neoplasms (13). In the present study, the functions and underlying mechanism of miR-375 in the regulation of CRC development were explored. miR-375 was found to inhibit CRC cell growth to some extent, and particularly suppress CRC cell migration and invasion ability. One study found that miR-375 could inhibit CRC cell growth by targeting PIK3CA (15), but there were no data documented concerning whether miR-375 could regulate CRC metastasis. The present study firstly found that miR-375 expression was negatively associated with metastasis, and also could suppress CRC cell migration and invasion ability. The functional research found that miR-375 expression in the metastatic CRC samples was less than that in the primary ones.

The molecular mechanisms underlying the function was further explored. SP1 was predicted and validated as a direct target of miR-375. SP1 is a ubiquitous nuclear transcription factor that regulates gene expression via multiple mechanisms (23), for example, by regulating cell cycle $(24,25)$, activating MMP2 (26) and promoting EMT process (27), to participate in the development of cell proliferation and invasion. In the present study, SP1 was verified as a direct target gene of miR-375. Thus, we predicted that miR-375 inhibited CRC development via targeting SP1.

In the present study, we found that miR-375 was significantly downregulated in CRC tissues from lymphoma and liver metastasis compared with the primary tumors. In addition, MMPs and the EMT process are important molecular events in cancer metastasis (28-31). In addition, we found that HCT8 cells had weak expression of miR-375, but higher invasion ability compared the DLD1, HCT116, HT29, LS174T cell lines stored in our laboratory, thus HCT8 was selected for further study of EMT (data not shown). EMT is a complicated process, with the most obvious feature: loss of E-cadherin and increased vimentin (32). In addition, genes such as snail, N-cadherin, $\beta$-catenin, are involved in the process (33-36). Further study was carried out to ascertain whether miR-375 could regulate MMP2 and EMT-associated genes. The results showed that overexpression of miR-375 inhibited the expression of SP1, MMP2, and also vimentin, snail, $\beta$-catenin, $\mathrm{N}$-cadherin, but increased E-cadherin, particularly on the cell membrane. In addition, the similar results were found when SP1 mRNA was interfered in CRC cells. Thus, we recognized that miR-375 is a key factor to inhibit CRC migration and invasion, by targeting SP1 through downregulating MMP2 and inhibiting the EMT process.

In conclusion, miR-375 inhibited proliferation, invasion and migration in CRC via directly targeting SP1 through 
EMT. In addition, miR-375 could be metastasis predictor and a treatment target for clinical application.

\section{References}

1. Gryfe R, Swallow C, Bapat B, Redston M, Gallinger S and Couture J: Molecular biology of colorectal cancer. Curr Probl Cancer 21: 233-300, 1997.

2. Jemal A, Bray F, Center MM, Ferlay J, Ward E and Forman D: Global cancer statistics. CA Cancer J Clin 61: 69-90, 2011.

3. Wittekind $\mathrm{C}$ and Neid $\mathrm{M}$ : Cancer invasion and metastasis. Oncology 69 (Suppl 1): S14-S16, 2005.

4. Fidler IJ, Yano S, Zhang RD, Fujimaki T and Bucana CD: The seed and soil hypothesis: Vascularisation and brain metastases. Lancet Oncol 3: 53-57, 2002.

5. Rudmik LR and Magliocco AM: Molecular mechanisms of hepatic metastasis in colorectal cancer. J Surg Oncol 92: 347-359, 2005.

6. Takayama T, Miyanishi K, Hayashi T, Sato Y and Niitsu Y: Colorectal cancer: Genetics of development and metastasis. J Gastroenterol 41: 185-192, 2006.

7. Ambros V: The functions of animal microRNAs. Nature 431: 350-355, 2004.

8. Bartel DP and Chen CZ: Micromanagers of gene expression: The potentially widespread influence of metazoan microRNAs. Nat Rev Genet 5: 396-400, 2004.

9. Bartel DP: MicroRNAs: Target recognition and regulatory functions. Cell 136: 215-233, 2009.

10. Lee Y, Ahn C, Han J, Choi H, Kim J, Yim J, Lee J, Provost P, Rådmark O, Kim S, et al: The nuclear RNase III Drosha initiates microRNA processing. Nature 425: 415-419, 2003.

11. Lund E, Güttinger S, Calado A, Dahlberg JE and Kutay U: Nuclear export of microRNA precursors. Science 303: 95-98, 2004.

12. Dai X, Chiang $\mathrm{Y}$, Wang $\mathrm{Z}$, Song $\mathrm{Y}, \mathrm{Lu} \mathrm{C}$, Gao $\mathrm{P}$ and $\mathrm{Xu} \mathrm{H}$ : Expression levels of microRNA-375 in colorectal carcinoma. Mol Med Rep 5: 1299-1304, 2012.

13. Wang S, Wang L, Bayaxi N, Li J, Verhaegh W, Janevski A Varadan V, Ren Y, Merkle D, Meng X, et al: A microRNA panel to discriminate carcinomas from high-grade intraepithelial neoplasms in colonoscopy biopsy tissue. Gut 62: 280-289, 2013.

14. Xu L, Li M, Wang M, Yan D, Feng G and An G: The expression of microRNA-375 in plasma and tissue is matched in human colorectal cancer. BMC Cancer 14: 714, 2014.

15. Wang Y, Tang Q, Li M, Jiang S and Wang X: MicroRNA-375 inhibits colorectal cancer growth by targeting PIK3CA. Biochem Biophys Res Commun 444: 199-204, 2014.

16. Lee YS and Dutta A: MicroRNAs in cancer. Annu Rev Pathol 4: 199-227, 2009

17. Le XF, Merchant O, Bast RC and Calin GA: The roles of microRNAs in the cancer invasion-metastasis cascade. Cancer Microenviron 3: 137-147, 2010.

18. Liu $\mathrm{M}$ and Chen $\mathrm{H}$ : The role of microRNAs in colorectal cancer. J Genet Genomics 37: 347-358, 2010.

19. El Ouaamari A, Baroukh N, Martens GA, Lebrun P, Pipeleers D and van ObberghenE: miR-375targets 3'-phosphoinositide-dependent protein kinase-1 and regulates glucose-induced biological responses in pancreatic beta-cells. Diabetes 57: 2708-2717, 2008

20. Poy MN, Hausser J, Trajkovski M, Braun M, Collins S, Rorsman P, Zavolan $M$ and Stoffel $M$ : miR-375 maintains normal pancreatic alpha- and beta-cell mass. Proc Natl Acad Sci USA 106: 5813-5818, 2009.
21. Ding L, Xu Y, Zhang W, Deng Y, Si M, Du Y, Yao H, Liu X, $\mathrm{Ke} \mathrm{Y}$, Si J, et al: MiR-375 frequently downregulated in gastric cancer inhibits cell proliferation by targeting JAK2. Cell Res 20: 784-793, 2010

22. Nishikawa E, Osada H, Okazaki Y, Arima C, Tomida S, Tatematsu Y, Taguchi A, Shimada Y, Yanagisawa K, Yatabe Y, et al: $m i R-375$ is activated by ASH1 and inhibits YAP1 in a lineage-dependent manner in lung cancer. Cancer Res 71: 6165-6173, 2011

23. Black AR, Black JD and Azizkhan-Clifford J: Sp1 and krüppellike factor family of transcription factors in cell grow th regulation and cancer. J Cell Physiol 188: 143-160, 2001.

24. Willoughby JA Sr, Sundar SN, Cheung M, Tin AS, Modiano J and Firestone GL: Artemisinin blocks prostate cancer growth and cell cycle progression by disrupting Sp1 interactions with the cyclin-dependent kinase-4 (CDK4) promoter and inhibiting CDK4 gene expression. J Biol Chem 284: 2203-2213, 2009.

25. Wei M, Liu B, Gu Q, Su L, Yu Y and Zhu Z: Stat6 cooperates with $\mathrm{Spl}$ in controlling breast cancer cell proliferation by modulating the expression of $\mathrm{p} 21^{\mathrm{Cip} 1 / \mathrm{WAF} 1}$ and $\mathrm{p} 27^{\mathrm{Kipl}}$. Cell Oncol 36: 79-93, 2013.

26. Chen Y, Huang Y, Huang Y, Xia X, Zhang J, Zhou Y, Tan Y, He S, Qiang F, Li A, et al: JWA suppresses tumor angiogenesis via Sp1-activated matrix metalloproteinase-2 and its prognostic significance in human gastric cancer. Carcinogenesis 35: 442-451, 2014.

27. Nam EH, Lee Y, Park YK, Lee JW and Kim S: ZEB2 upregulates integrin $\alpha 5$ expression through cooperation with $\mathrm{Spl}$ to induce invasion during epithelial-mesenchymal transition of human cancer cells. Carcinogenesis 33: 563-571, 2012.

28. Hay ED: An overview of epithelio-mesenchymal transformation. Acta Anat 154: 8-20, 1995.

29. Kalluri R and Weinberg RA: The basics of epithelial-mesenchymal transition. J Clin Invest 119: 1420-1428, 2009.

30. Zhu QC, Gao RY, Wu W and Qin HL: Epithelial-mesenchymal transition and its role in the pathogenesis of colorectal cancer. Asian Pac J Cancer Prev 14: 2689-2698, 2013.

31. Said AH, Raufman JP and Xie G: The role of matrix metalloproteinases in colorectal cancer. Cancers 6: 366-375, 2014.

32. Huber MA, Kraut N and Beug H: Molecular requirements for epithelial-mesenchymal transition during tumor progression. Curr Opin Cell Biol 17: 548-558, 2005.

33. Kim K, Lu Z and Hay ED: Direct evidence for a role of betacatenin/LEF-1 signaling pathway in induction of EMT. Cell Biol Int 26: 463-476, 2002 .

34. Nakajima S, Doi R, Toyoda E, Tsuji S, Wada M, Koizumi M, Tulachan SS, Ito D, Kami K, Mori T, et al: N-cadherin expression and epithelial-mesenchymal transition in pancreatic carcinoma. Clin Cancer Res 10: 4125-4133, 2004.

35. Zavadil J, Cermak L, Soto-Nieves $\mathrm{N}$ and Böttinger EP: Integration of TGF-beta/Smad and Jagged1/Notch signalling in epithelial-to-mesenchymal transition. EMBO J 23: 1155-1165, 2004.

36. Medici D, Hay ED and Olsen BR: Snail and Slug promote epithelial-mesenchymal transition through beta-catenin-T-cell factor-4-dependent expression of transforming growth factorbeta3. Mol Biol Cell 19: 4875-4887, 2008. 